\title{
Serum neurofilament light chain is not a useful biomarker of central nervous system involvement in women with Fabry disease
}

\author{
Tomasz Hołub, Kamila Kędzierska, Katarzyna Muras-Szwedziak, Michał Nowicki*
}

Department of Nephrology, Hypertension and Kidney Transplantation, Medical University of Lodz, Poland.

SUMMARY Neurofilament Light Chain (NfL) serum concentration is a new noninvasive marker of neurodegenerative disorders. Fabry disease (FD) leads to accumulation of glycosphingolipids in tissues leading to progressive damage of critical body systems and organs, including peripheral and central nervous system. There are no established serum markers of neurodegeneration in FD. Our crosssectional single-center study was designed to prove the concept that serum NfL levels could reflect the severity of cognitive impairment and indirectly, the level of central nervous system involvement in women at earlier stages of FD. Twelve women with a diagnosis of FD confirmed by genetic tests and 12 matched healthy subjects were included. Serum concentrations of NfL were measured in all subjects together with neuropsychological tests that included Mini Mental State Examination (MMSE) and Montreal Cognitive Assessment Scale (MoCA). Quality of life was assessed with the Short Form Survey (SF-36). FD patients and healthy subjects did not differ with respect to serum NfL concentration, results of neuropsychological tests and quality of life. There was a significant positive correlation between NfL and globotriaosylosphingosine (lyso-Gb3) concentration in women with FD $(\mathrm{R}=0,69, p=0.01)$. There was also a correlation between NfL concentration and MoCA score but not MMSE score. Receiver operating characteristic (ROC) analysis showed that the best predictor for Mild Cognitive Impairment in both groups was eGFR. Serum NfL concentration does not appear to predict the degree of nervous system involvement in women with FD.

Keywords biomarker, neurodegeneration, quality of life

\section{Introduction}

Fabry disease (FD), is an ultra-rare lysosomal storage disease inherited as an X-linked disorder. FD is caused by a deficiency of the lysosomal enzyme alphagalactosidase A ( $\alpha$-Gal A; E.C. 3.2.1.22). GLA gene, located on X chromosome at Xq22, encodes a 429 amino acid precursor that is processed to a 398 amino acid glycoprotein functioning as a homodimer. The mutation of GLA leads to a deficiency or absence of the enzyme $\alpha$-galactosidase A ( $\alpha$-Gal A), which catalyzes the hydrolysis of globotriaosylceramide.

Alpha-Gal A deficiency leads to progressive accumulation of glycolipids, and globotriaosylosphingosine (lyso-Gb3) in different cells of the body, leading to damage and dysfunction of affected organs. The most affected cells and tissues in FD include glomerular podocytes, cardiomyocytes, endothelial cells, vascular muscles and peripheral and central nerves. It all leads to dysfunction and failure of vital organs including the heart, kidneys and nervous system. The severity of the disease depends on the gender, age and the type of mutation. Males with classic phenotype have the highest risk of complications and early symptoms, while younger women mostly become affected by FD later in life (1).

Neurofilaments are the main component of the neuronal cytoskeleton. Light (NfL), intermediate (NfM) and heavy $(\mathrm{NfH})$ chains have been distinguished on the basis of their molecular mass. The serum concentration of NfL and their importance as a marker of central nervous system diseases have been demonstrated in several recent studies $(2,3)$. Neuronal damage and physiological changes of the central nervous system (CNS) cause release of neurofilaments. This translates into elevated levels of NfL in the cerebrospinal fluid and ultimately in the blood, where its concentration reflects the rate at which $\mathrm{NfL}$ is released from the neurons (3). Several studies have shown a strong positive correlation between NfL in the blood and in the cerebrospinal fluid (4). Serum NfL concentration positively correlated with severity of various diseases of the central nervous system including multiple sclerosis, amyotrophic lateral sclerosis, frontotemporal 
dementia, Alzheimer's disease, traumatic brain injuries and degeneration of the nervous system associated with HIV infection (2).

The main physiologic factor influencing the NfL concentration is the age of the patients. With aging NfL concentration in healthy subjects increased by $2.2 \%$ annually. After the age of 60 , a further significant increase in NfL concentration is observed (5). These changes could be attributed both to aging itself and aggregation of comorbidities. It has been well proven that patients with FD are characterized with much faster brain aging compared to the healthy population (6). Patients with FD are at increased risk of developing cognitive dysfunction and most of them also have symptoms of depression (7). Patients diagnosed with severe depression have more cognitive impairment compared to the general population (8). It also was shown that in major depressive disorder higher levels of NfL were observed (9). However, it has not been confirmed in FD that the cognitive impairment is due to depressive symptoms but its risk factors include male gender, patients with classic disease phenotype, lower intelligence quotient (IQ) and a history of stroke $(10,11)$.

Many clinical tests have been developed to assess cognitive functions, each of which assesses specific domains of cognitive functioning, but in a different aspect. Screening tests play a key role in allowing each clinician to perform an initial assessment of cognitive impairment. The best validated tests used for screening for cognitive impairment include the Mini Mental State Examination (MMSE) and the Montreal Cognitive Assessment Scale (MoCA) (12,13). MMSE and MoCA were used and well-validated in recent studies assessing cognitive impairment in patients with $\operatorname{FD}(11,14)$.

The aim of the study was to assess whether the serum concentration of NfL could be a marker of the early central nervous system involvement and cognitive impairment in women with FD.

\section{Materials and Methods}

The study was approved by the Local Ethics Committee and was conducted in accordance with the Declaration of Helsinki. All patients gave informed written consent to participate in the study.

Twenty-four subjects were enrolled, including 12 women with confirmed FD and 12 matched healthy controls. The study was conducted between March and October 2020. The characteristics of the study population is provided in Table 1. Diagnosis of FD was based on the blood concentration of $\alpha-G a l$ A, lyso-Gb3 and on genetic tests. The tests were performed using the Dry Blood Spot method (DBS). Individual results are presented in Table 2. Only one woman from the study group has been qualified for enzyme replacement therapy.

The patients with FD included in our study came from three different families. The degree of kindship and

Table 1. Clinical characteristics of the study population

\begin{tabular}{|c|c|c|c|c|c|c|c|c|}
\hline No. & Age & eGFR & Education & MMSE & $\mathrm{MoCA}$ & SF-36 & Diabetes & Stroke \\
\hline \multicolumn{9}{|c|}{ Fabry disease group } \\
\hline 1 & 42 & 88.8 & 12 & 28 & 28 & 62 & No & No \\
\hline 2 & 21 & 129.5 & 12 & 26 & 29 & 28 & No & No \\
\hline 3 & 64 & 45.8 & 8 & 24 & 24 & 87 & Yes & Yes \\
\hline 4 & 42 & 91.6 & 17 & 30 & 29 & 62 & No & No \\
\hline 5 & 45 & 113.1 & 17 & 30 & 29 & 23 & No & No \\
\hline 6 & 71 & 32.4 & 12 & 30 & 24 & 74 & No & Yes \\
\hline 7 & 51 & 63.5 & 17 & 25 & 25 & 19 & No & No \\
\hline 8 & 22 & 127.9 & 12 & 30 & 29 & 28 & No & No \\
\hline 9 & 42 & 111.5 & 17 & 28 & 27 & 27 & No & No \\
\hline 10 & 47 & 94.7 & 17 & 28 & 25 & 35 & No & No \\
\hline 11 & 20 & 133.7 & 12 & 30 & 30 & 15 & No & No \\
\hline 12 & 40 & 110.7 & 17 & 30 & 28 & 39 & No & No \\
\hline \multicolumn{9}{|c|}{ Control group } \\
\hline 1 & 42 & 116.2 & 17 & 30 & 30 & 18 & No & No \\
\hline 2 & 68 & 95.3 & 12 & 25 & 23 & 63 & No & No \\
\hline 3 & 36 & 116.3 & 13 & 26 & 26 & 52 & No & No \\
\hline 4 & 30 & 108.7 & 20 & 30 & 30 & 26 & No & No \\
\hline 5 & 37 & 109.7 & 20 & 30 & 30 & 23 & No & No \\
\hline 6 & 53 & 94.4 & 17 & 30 & 26 & 22 & No & No \\
\hline 7 & 48 & 129.7 & 17 & 30 & 30 & 44 & No & No \\
\hline 8 & 54 & 68.7 & 12 & 26 & 24 & 67 & Yes & No \\
\hline 9 & 41 & 42 & 12 & 29 & 28 & 74 & No & No \\
\hline 10 & 76 & 34.4 & 12 & 24 & 19 & 84 & Yes & No \\
\hline 11 & 73 & 46 & 12 & 29 & 24 & 100 & No & No \\
\hline 12 & 54 & 68.7 & 12 & 26 & 24 & 67 & Yes & No \\
\hline
\end{tabular}

eGFR: estimated Glomerular Filtration Rate; MMSE: Mini Mental State Examination; MoCA: Montreal Cognitive Assessment Scale; SF-36:The Short Form 36 Health Survey. 
Table 2. Type of genetic variant, globotriaosylosphingosine and $\alpha$-galactosidase $A$ concentration in blood in women with Fabry disease

\begin{tabular}{|c|c|c|c|}
\hline $\begin{array}{l}\text { Patients } \\
\text { No }\end{array}$ & Mutation & $\begin{array}{c}\text { Lyso-Gb3 } \\
{[\mathrm{ng} / \mathrm{mL}]}\end{array}$ & $\begin{array}{c}\alpha-\mathrm{Gal} \mathrm{A} \\
{[\mu \mathrm{mol} / \mathrm{L} / \mathrm{h}]}\end{array}$ \\
\hline 1 & c. $680 \mathrm{G}>\mathrm{A}(\mathrm{p} . \operatorname{Arg} 227 \mathrm{Gln})$ & 4.6 & 4.7 \\
\hline 2 & c. $680 \mathrm{G}>\mathrm{A}(\mathrm{p} . \mathrm{Arg} 227 \mathrm{Gln})$ & 4.4 & 1.4 \\
\hline 3 & c. $439 \mathrm{~g}>\mathrm{A}$ (p.Gly147Arg) & 6.8 & 1.7 \\
\hline 4 & c. $439 \mathrm{~g}>\mathrm{A}$ (p.Gly147Arg) & 8 & 1.9 \\
\hline 5 & c.439g $>$ A (p.Gly147Arg) & 10.2 & 3.3 \\
\hline 6 & $\begin{array}{l}\text { c. } 138 \mathrm{C}>\mathrm{G} \text { (p.His46Gln) } \\
\text { c. } 153 \mathrm{G}>\mathrm{T} \text { (p.Met51Ile) } \\
\text { c.167G }>\mathrm{T} \text { (p.Cys56Phe) }\end{array}$ & 22.7 & 0.4 \\
\hline 7 & $\begin{array}{l}\text { c. } 138 \mathrm{C}>\mathrm{G}(\mathrm{p} . \text { His } 46 \mathrm{Gln}) \\
\mathrm{c} .153 \mathrm{G}>\mathrm{T} \text { (p.Met51Ile) } \\
\text { c. } 167 \mathrm{G}>\mathrm{T} \text { (p.Cys56Phe) }\end{array}$ & 12.8 & 1.2 \\
\hline 8 & $\begin{array}{l}\text { c. } 138 \mathrm{C}>\mathrm{G}(\mathrm{p} . \text { His } 46 \mathrm{Gln}) \\
\mathrm{c} .153 \mathrm{G}>\mathrm{T} \text { (p.Met51Ile) } \\
\text { c.167G }>\mathrm{T} \text { (p.Cys56Phe) }\end{array}$ & 4.6 & 3 \\
\hline 9 & $\begin{array}{l}\text { c. } 138 \mathrm{C}>\mathrm{G}(\mathrm{p} . \text { His } 46 \mathrm{Gln}) \\
\mathrm{c} .153 \mathrm{G}>\mathrm{T} \text { (p.Met51Ile) } \\
\text { c.167G }>\mathrm{T} \text { (p.Cys56Phe) }\end{array}$ & 5.2 & 1.6 \\
\hline 10 & $\begin{array}{l}\text { c. } 138 \mathrm{C}>\mathrm{G}(\mathrm{p} . \text { His } 46 \mathrm{Gln}) \\
\mathrm{c} .153 \mathrm{G}>\mathrm{T} \text { (p.Met51Ile) } \\
\text { c. } 167 \mathrm{G}>\mathrm{T} \text { (p.Cys56Phe) }\end{array}$ & 11 & 2.4 \\
\hline 11 & $\begin{array}{l}\text { c. } 138 \mathrm{C}>\mathrm{G}(\mathrm{p} . \text { His } 46 \mathrm{Gln}) \\
\mathrm{c} .153 \mathrm{G}>\mathrm{T} \text { (p.Met51Ile) } \\
\text { c. } 167 \mathrm{G}>\mathrm{T} \text { (p.Cys56Phe) }\end{array}$ & 7.1 & 1.8 \\
\hline 12 & $\begin{array}{l}\text { c. } 138 \mathrm{C}>\mathrm{G} \text { (p.His46Gln) } \\
\text { c. } 153 \mathrm{G}>\mathrm{T} \text { (p.Met51Ile) } \\
\text { c.167G }>\mathrm{T} \text { (p.Cys56Phe) }\end{array}$ & 23.9 & 0.6 \\
\hline
\end{tabular}

Lyso-Gb3: globotriaosylosphingosine; $\alpha$-Gal A - $\alpha$-galactosidase A.

family trees of the patients are presented in Figure 1.

The control group consisted of 12 healthy women, matched to women with FD for age, education level and kidney function. The exclusion criteria were the diagnosis of any disease of the central nervous system other than associated with FD, a disability that would hinder any of the study procedures such as hearing or vision loss, chronic kidney disease with eGFR $<30 \mathrm{~mL} /$ min, dementia, acute functional psychiatric disorder and uncontrolled hypertension (systolic BP $>130 \mathrm{mmHg}$ or diastolic $\mathrm{BP}>80 \mathrm{mmHg}$ ).

Every patient completed a quality-of-life questionnaire The Short Form 36 Health Survey (SF-36) and two screening tests assessing cognitive function, MoCA and MMSE.

The MoCA and MMSE are commonly used as the screening neuropsychological scales. In MMSE, the most commonly used cutoff point for the diagnosis of dementia is a score of 24 points or less. The maximum score for the MoCA test is 30 points; a result of 26 or more points is defined as normal. A score from 19 to 25 is considered as mild cognitive impairment (MCI) (13).

During the same visit blood was collected in a fasting state after an overnight rest from all participants to determine serum concentration of NfL, creatinine, urea, calcium, phosphate, parathyroid hormone (PTH) and blood hemoglobin. The concentration of NfL was
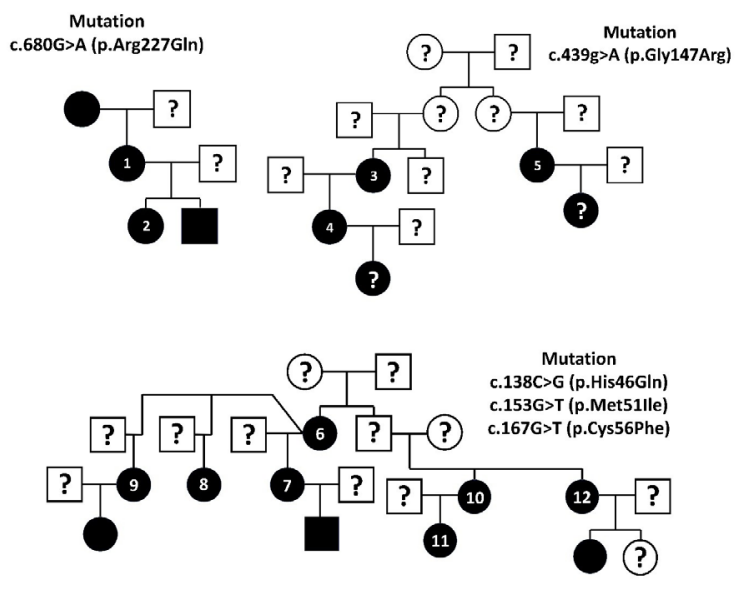

Figure 1. Family trees of three families with Fabry disease included in our study.

assessed with a Neurofilament Light Polypeptide (NEFL) ELISA Kit (antibodies-online GmbH, Aachen, Germany). Other parameters were measured using routine automated laboratory methods in a local laboratory.

The mean value and standard deviation were calculated for each normally distributed variable. For non-normally distributed variables median value with the interquartile range (IQR) was calculated. Analysis of the normality of the distribution was performed with the Shapiro-Wilk test, and on the basis of its results, the parametric $t$-test or the non-parametric Mann-Whitney $U$ test was used. Receiver operating characteristic (ROC) curves were drawn to assess the value of serum concentration of NfL, eGFR, lyso-Gb3 and $\alpha$-Gal A indicating presence of mild cognitive impairment in the MoCA test. Statistica 13.1 software was used to perform the statistical analysis.

\section{Results and Discussion}

Serum NfL concentration did not significantly differ between groups $(0,053 \pm 0,1$ vs. $0,048 \pm 0,09 \mathrm{ng} /$ $\mathrm{mL} ; p=0.9)$. Women with FD also had similar blood hemoglobin, serum phosphate and PTH. Serum calcium concentration was higher in the FD group than in healthy women $(2.38 \pm 0.08$ vs. $2.28 \pm 0.11 \mathrm{mmoL} /$ $\mathrm{L}$, respectively; $p=0.03$ ). MMSE, MoCA and SF-36 scores were also similar in each group.

In women with FD there was a significant positive correlation between age and serum PTH concentration $(\mathrm{R}=0.62, p=0.03)$. The same correlation was not seen in the control group.

In the FD group there was also a significant positive correlation between $\mathrm{NfL}$ and lyso-Gb3 concentration ( $\mathrm{R}$ $=0.69, p=0.01$ ).

In the control group a significant negative correlation was found between serum NfL and hemoglobin $(\mathrm{R}=$ $-0.8, p=0.001)$ MoCA score $(\mathrm{R}=-0.6, p=0.04)$, and a positive correlation between NfL and serum PTH 

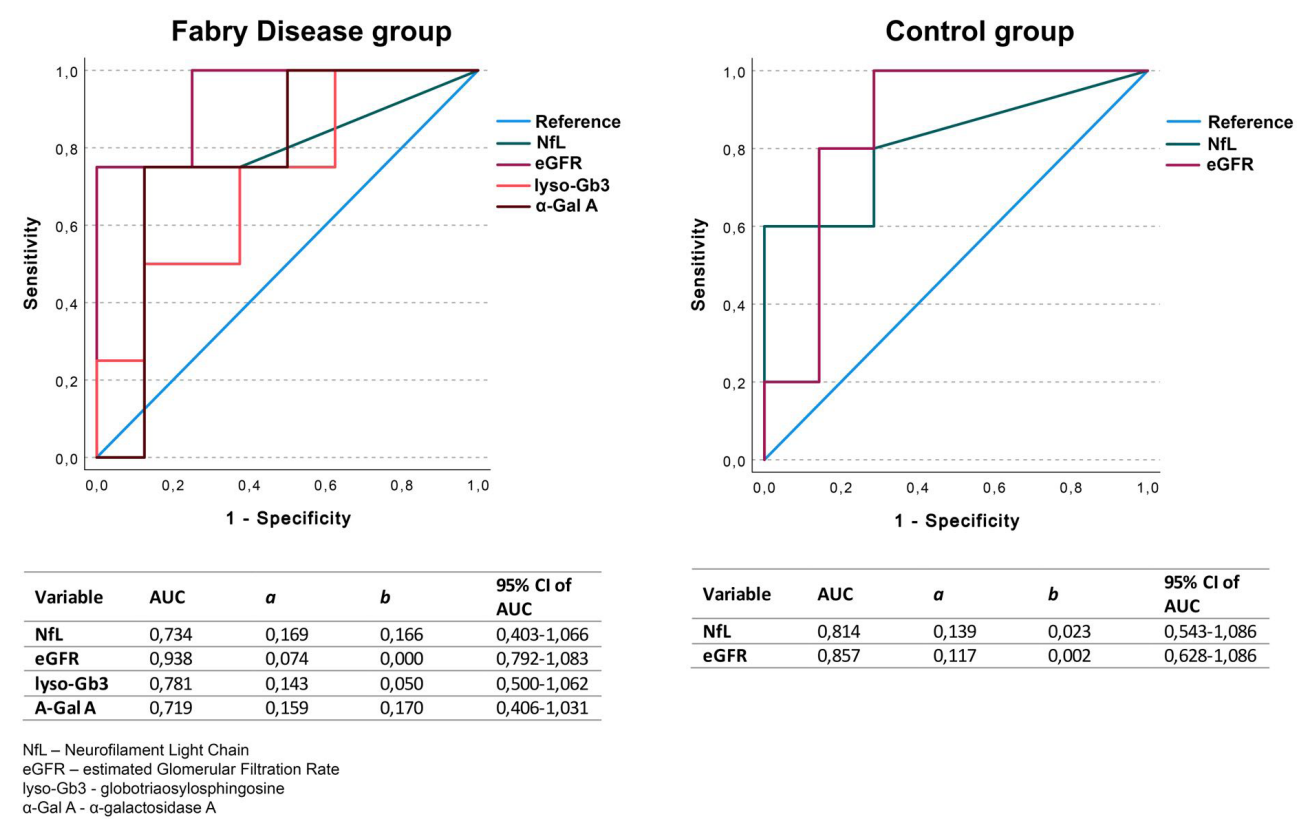

Figure 2. Predictors of mild cognitive impairment and receiver operating characteristic curves.

concentration $(\mathrm{R}=0.8, p=0.0009)$. These correlations were not present in women with FD.

In the control group there was a strong negative correlation between age and MoCA score $(\mathrm{R}=-0.83$, $p=0.0009)$ and a positive correlation between age and SF-36 score $(\mathrm{R}=0.6, p=0.04)$. In the FD group only a significant correlation between age and MoCA score was observed $(\mathrm{R}=-0.85, p=0.0004)$.

$\mathrm{ROC}$ analysis showed that the best predictor for $\mathrm{MCI}$ in both groups was eGFR. Area under the curve (AUC) for women with FD was 0.938 (95\% CI: $0.792-1.083)$ and in the control group 0.857 (95\% CI: $0.628-1.086)$. Detailed information on ROC analysis results is provided in Figure 2.

Our cross-sectional single-center study was designed to prove the concept that serum NfL levels could reflect the severity of cognitive impairment and indirectly, the level of CNS involvement in women at earlier stages of FD without previous clinical symptoms of nervous system involvement. The hypothesis behind the study was that damage to the neurons in FD patients will result in an increase in concentration of NfL in cerebrospinal fluid and its penetration into the peripheral circulation, which can be assessed by measuring their serum levels. Loeffler $\mathrm{T}$, et al. in a mouse model showed that NfL concentration can be a valuable biomarker not only in typical neurodegenerative diseases but also in other diseases that have an additional neuronal component, like lysosomal storage diseases, e.g., Gaucher disease (15). Erante D, et al. confirmed that NfL is a good biomarker of neurodegeneration in Niemann-Pick disease (16). Ru Y, et al. described NfL as a biomarker of neurodegeneration in neuronal ceroid lipofuscinosis type
2 (CLN2 disease), another lysosomal storage disease. These authors showed in a canine model a significant correlation between serum NfL concentration and disease progression. Differently, in the human part of their study they were unable to show correlations between NfL concentration and CLN2 severity or age of the patients. However they showed, that NfL concentration decreased by $50 \%$ after enzyme replacement therapy was initiated (17).

The above cited studies assessed the utility of NfL as a biomarker for the nervous system involvement in some lysosomal storage diseases. To our knowledge, there have been no similar studies in patients with FD. In FD women usually have a milder disease course than men, which is due to a random inactivation of the $\mathrm{X}$ chromosome. However, severe involvement of such target organs as the heart or kidneys is quite common (18). Despite residual enzyme activity, women with FD develop characteristic symptoms with age, including central nervous system symptoms. However, the clinical manifestation is more varied and the symptoms appear about 10 years later compared to men. The median interval between the onset of early FD symptoms and correct diagnosis is even more delayed in women, with a delay of 19 years on average (19). The identification of the marker of early nervous system involvement in women with FD could be particularly clinically relevant since women with this disease develop symptoms much later than men but the nervous system is most frequently involved. The symptoms of FD significantly interfere with patients daily functioning, which contributes to a significantly reduced lifespan. Studies show that the life expectancy of men with Fabry disease is 15 to even 20 
years shorter, and that of women is 6 to 10 years shorter compared to the average life expectancy in the population (20). In our study, we were not able to confirm that NfL concentration is a clinically useful biomarker for an assessment of the degree of nervous system involvement in women with FD. It may be due to the young age of the patients included in the study and the fact that most of them had no or moderate typical symptoms of the disease from other organs than the nervous system. In most of our study subjects genetic tests were performed due to diagnosis of FD in their relatives.

The $\alpha$-Gal activities and the lyso-Gb3 concentration are the serum markers commonly used in the diagnosis and monitoring of FD. Measurement of $\alpha$-Gal activity in plasma or leukocytes, which is the reference method for laboratory confirmation of the diagnosis in men, is often inconclusive in female patients whose enzymatic activity can range from low to normal values. In our study $25 \%$ of women with FD had normal $\alpha$-Gal activity and the lyso-Gb3 concentration was above normal in all cases. The basis underlying variability of the phenotype in women is still poorly understood, but the role of $\mathrm{X}$ chromosome inactivation appears to be most important (21). In FD, lyso-Gb3 levels are always elevated in men, but only between 40 and $60 \%$ in women. Lyso-Gb3 levels in women increase with age and are within the normal range in childhood. However, when symptomatic FD is suspected in adult women, both measurement of $\alpha$-Gal A and plasma lyso-Gb3 activity improves the diagnostic value (18).

In our study the concentration of lyso-Gb3 did not correlate with the results of the tests validated for diagnosis of cognitive dysfunction and with the results of the SF-36. Despite no difference in NfL concentration between study and control group, a positive significant correlation between the concentration of lyso-Gb3 and $\mathrm{NfL}$ in the populations of women with FD was seen. An accumulation of lyso-Gb3 deposits as a result of $\alpha-G a l$ A deficiency causes damage to nerve cells with subsequent pro-inflammatory activity $(22,23)$. It is possible that due to early diagnosis of the disease and lack of previous neurologic symptoms in our study group, the risk of neurodegeneration was low and thus no difference in serum NfL concentration between the two groups was observed.

Studies conducted so far have shown that patients with FD have significant cognitive deficits (24). Most of the studies attributed the changes of cognitive functioning in the course of FD to the accumulation of glycosphingolipids in the cerebral circulation (25). In our study, however, no relationship was found between lyso-Gb3 concentration and cognitive impairment. Many psychological tests have been developed for screening for cognitive impairment in clinical practice. They differ in their sensitivity and specificity. The most commonly used screening test in the diagnosis of cognitive impairment is MMSE. Körver S, et al. showed that MMSE did not allow screening for MCI in patients with FD and it may lose its predictive value when the cognitive impairment is milder, less prevalent and occurs predominantly in the executive domain, as appears to be the case in FD (14). Our results are consistent with the finding that MMSE cannot accurately distinguish patients with subtle cognitive impairment from patients without clinically detectable cognitive impairment. Therefore MoCA should be preferred to MMSE in populations at risk for MCI or with early-stage dementia. In a study conducted by Körver S, et al. MoCA questionnaire classified 21\% of patients with FD as having MCI, compared with 11\% in the reference group (14). These data are consistent with our results. In our study more patients were identified as having MCI with the MoCA test compared to MMSE.

In our study, the mean score in MMSE and MoCA test was similar in FD women and control subjects. Löhle $\mathrm{M}$, et al. also did not find any significant difference in the tests accessing cognitive functions between the patients with FD and healthy subjects. Their study group was larger, included both women and men in different stages of FD and with both classic and late type (11). In contrast our study included only women without any signs of central nervous system involvement.

Our study results indicate that neither depression nor disease severity, time since FD symptoms, and enzyme activity predicted a cognitive dysfunction. The analysis found no association between cognitive impairment and test scores.

Kidney disease is one of the major complications of FD and is associated with a continuous accumulation of glycosphingolipids throughout the nephron. This leads to progressive loss of GFR and eventually to renal failure. The kidney and brain share similar hemodynamic abilities, such as vasoregulation of the microcirculation. Studies have shown that lower eGFR was associated with increasing severity of chronic white matter hyperintensities (CWMH), and patients with more stable eGFR had fewer strokes than those with rapidly progressive kidney disease (26). Our study also suggests the importance of eGFR levels as a predictor of MCI in this patient group. Therefore, further research into the relationship between eGFR levels and MCI in FD patients is warranted (9).

In our study, we did not show an association between elevated serum calcium levels in women with FD and serum PTH. In one previous study the authors tried to elucidate the pathomechanism of calcium-phosphate disturbances in patients with FD using a mouse model GlatmTg (CAG-A4GALT). The study results showed a relationship between hypercalcemia and hypercalciuria and secondary hyperthyroidism (27). This may suggest that patients with FD are at increased risk of accelerated bone resorption and osteomalacia (28).

Our study has limitations, because we have assessed the concentration of NfL only in the peripheral 
circulation. However, previous studies showed that the concentration of NfL in the blood strongly correlates with its concentration in the cerebrospinal fluid $(29,30)$. Another limitation is a small study group, which is a result of the ultra-low incidence of the disease in the population and of the selection of only the female patients without any previous signs of central nervous system involvement typical for FD.

\section{Conclusions}

The results of our study did not confirm the relation between the degree of nervous system involvement in women with FD and serum NfL levels. Therefore serum NfL cannot be considered as a useful marker of cognitive impairment in this disease. Our study also showed that MoCA is the preferred test to detect mild cognitive impairment in FD.

Funding: The study was supported by the Medical University of Lodz grant No. 503/1-151-02/503-01.

Conflict of Interest: The authors have no conflicts of interest to disclose.

\section{References}

1. Sodré LSS, Huaira RMNH, Bastos MG, Colugnati FAB, Coutinho MP, Fernandes NMDS. Screening for Fabry disease in kidney disease: a cross-sectional study in males and females. Kidney Blood Press Res. 2017; 42:12581265.

2. Weydt P, Oeckl P, Huss A, Muller K, Volk AE, Kuhle J, Knehr A, Andersen PM, Prudlo J, Steinacker P, Weishaupt JH, Ludolph AC, Otto M. Neurofilament levels as biomarkers in asymptomatic and symptomatic familial amyotrophic lateral sclerosis. Ann Neurol. 2016; 79:152158.

3. Khalil M, Teunissen CE, Otto M, Piehl F, Sormani MP, Gattringer T, Barro C, Kappos L, Comabella M, Fazekas F, Petzold A, Blennow K, Zetterberg H, Kuhle J. Neurofilaments as biomarkers in neurological disorders. Nat Rev Neurol. 2018; 14:577-589.

4. Thebault S, D RT, Lee H, Bowman M, Bar-Or A, Arnold DL, H LA, Tabard-Cossa V, Freedman MS. High serum neurofilament light chain normalizes after hematopoietic stem cell transplantation for MS. Neurol Neuroimmunol Neuroinflamm. 2019; 6:e598.

5. Khalil M, Pirpamer L, Hofer E, Voortman MM, Barro C, Leppert D, Benkert P, Ropele S, Enzinger C, Fazekas F, Schmidt R, Kuhle J. Serum neurofilament light chain levels in normal aging and their association with morphologic brain changes. Nat Commun. 2020; 11:812.

6. Wadley VG, McClure LA, Warnock DG, Lassen-Greene CL, Hopkin RJ, Laney DA, Clarke VM, Kurella Tamura M, Howard G, Sims K. Cognitive function in adults aging with fabry disease: a case-control feasibility study using telephone-based assessments. JIMD Rep. 2015; 18:41-50.

7. Cole AL, Lee PJ, Hughes DA, Deegan PB, Waldek S, Lachmann RH. Depression in adults with Fabry disease: a common and under-diagnosed problem. J Inherit Metab Dis. 2007; 30:943-951.

8. Rock PL, Roiser JP, Riedel WJ, Blackwell AD. Cognitive impairment in depression: a systematic review and metaanalysis. Psychol Med. 2014; 44:2029-2040.

9. Tapia D, Kimonis V. Stroke and chronic kidney disease in Fabry disease. J Stroke Cerebrovasc Dis. 2021; 30:105423.

10. Loeb J, Feldt-Rasmussen U, Madsen CV, Vogel A. Cognitive impairments and subjective cognitive complaints in Fabry disease: a nationwide study and review of the literature. JIMD Rep. 2018; 41:73-80.

11. Lohle M, Hughes D, Milligan A, Richfield L, Reichmann H, Mehta A, Schapira AH. Clinical prodromes of neurodegeneration in Anderson-Fabry disease. Neurology. 2015; 84:1454-1464.

12. Tombaugh TN, McIntyre NJ. The mini-mental state examination: a comprehensive review. J Am Geriatr Soc. 1992; 40:922-935.

13. Zhuang L, Yang Y, Gao J. Cognitive assessment tools for mild cognitive impairment screening. J Neurol. 2021; 268:1615-1622.

14. Körver S, van de Schraaf SAJ, Geurtsen GJ, Hollak CEM, van Schaik IN, Langeveld M. The mini mental state examination does not accurately screen for objective cognitive impairment in Fabry Disease. JIMD Rep. 2019; 48:53-59.

15. Loeffler T, Schilcher I, Flunkert S, Hutter-Paier B. Neurofilament-light chain as biomarker of neurodegenerative and rare diseases with high translational value. Front Neurosci. 2020; 14:579.

16. Eratne D, Loi SM, Li QX, Varghese S, McGlade A, Collins S, Masters CL, Velakoulis D, Walterfang M. Cerebrospinal fluid neurofilament light chain is elevated in Niemann-Pick type $\mathrm{C}$ compared to psychiatric disorders and healthy controls and may be a marker of treatment response. Aust N Z J Psychiatry. 2020; 54:648-649.

17. Ru Y, Corado C, Soon RK, Jr, Melton AC, Harris A, Yu GK, Pryer N, Sinclair JR, Katz ML, Ajayi T, Jacoby D, Russell CB, Chandriani S. Neurofilament light chain is a treatment-responsive biomarker in CLN2 disease. Ann Clin Transl Neurol. 2019; 6:2437-2447.

18. Michaud M, Mauhin W, Belmatoug N, Garnotel R, Bedreddine N, Catros F, Ancellin S, Lidove O, Gaches F. When and how to diagnose Fabry disease in clinical pratice. Am J Med Sci. 2020; 360:641-649.

19. Godel T, Köhn A, Muschol N, Kronlage M, Schwarz D, Kollmer J, Heiland S, Bendszus M, Mautner VF, Baumer P. Dorsal root ganglia in vivo morphometry and perfusion in female patients with Fabry disease. J Neurol. 2018; 265:2723-2729.

20. Nowicki M, Bazan-Socha S, Blazejewska-Hyzorek B, Gellert R, Imiela J, Kazmierczak J, Klopotowski M, OkoSarnowska Z, Pawlaczyk K, Ponikowski P, Slawek J, Sykut-Cegielska J, Witkowski A, Zwolinska D. Enzyme replacement therapy in Fabry disease in Poland: a position statement. Pol Arch Intern Med. 2020; 130:91-97.

21. Echevarria L, Benistan K, Toussaint A, Dubourg O, Hagege AA, Eladari D, Jabbour F, Beldjord C, De Mazancourt P, Germain DP. X-chromosome inactivation in female patients with Fabry disease. Clin Genet. 2016; 89:44-54.

22. De Francesco PN, Mucci JM, Ceci R, Fossati CA, Rozenfeld PA. Higher apoptotic state in Fabry disease peripheral blood mononuclear cells.: effect of 
globotriaosylceramide. Mol Genet Metab. 2011; 104:319324.

23. De Francesco PN, Mucci JM, Ceci R, Fossati CA, Rozenfeld PA. Fabry disease peripheral blood immune cells release inflammatory cytokines: role of globotriaosylceramide. Mol Genet Metab. 2013; 109:9399.

24. Elstein D, Doniger GM, Altarescu G. Cognitive testing in Fabry disease: pilot using a brief computerized assessment tool. Isr Med Assoc J. 2012; 14:624-628.

25. Ferraz MJ, Kallemeijn WW, Mirzaian M, Herrera Moro D, Marques A, Wisse P, Boot RG, Willems LI, Overkleeft HS, Aerts JM. Gaucher disease and Fabry disease: new markers and insights in pathophysiology for two distinct glycosphingolipidoses. Biochim Biophys Acta. 2014; 1841:811-825.

26. Oksala NK, Salonen T, Strandberg T, Oksala A, Pohjasvaara T, Kaste M, Karhunen PJ, Erkinjuntti T. Cerebral small vessel disease and kidney function predict long-term survival in patients with acute stroke. Stroke. 2010; 41:1914-1920.

27. Maruyama H, Taguchi A, Mikame M, Lu H, Tada N, Ishijima M, Kaneko H, Kawai M, Goto S, Saito A, Ohashi R, Nishikawa Y, Ishii S. Low bone mineral density due to secondary hyperparathyroidism in the Gla(tm) $\mathrm{Tg}(\mathrm{CAG}-$ A4GALT) mouse model of Fabry disease. FASEB Bioadv. $2020 ; 2: 365-381$.
28. Germain DP, Benistan K, Boutouyrie P, Mutschler C. Osteopenia and osteoporosis: previously unrecognized manifestations of Fabry disease. Clin Genet. 2005; 68:9395.

29. Novakova L, Zetterberg H, Sundström P, Axelsson M, Khademi M, Gunnarsson M, Malmeström C, Svenningsson A, Olsson T, Piehl F, Blennow K, Lycke J. Monitoring disease activity in multiple sclerosis using serum neurofilament light chain protein. Neurology. 2017; 89:2230-2237.

30. Bavato F, Cathomas F, Klaus F, Gütter K, Barro C, Maceski A, Seifritz E, Kuhle J, Kaiser S, Quednow BB. Altered neuroaxonal integrity in schizophrenia and major depressive disorder assessed with neurofilament light chain in serum. J Psychiatr Res. 2021; 140:141-148.

Received August 11, 2021; Revised October 27, 2021; Accepted November 2, 2021.

*Address correspondence to:

Michał Nowicki, Department of Nephrology, Hypertension and Kidney Transplantation, Central University Hospital, Medical University od Lodz, ul. Pomorska 251, 92-213 Lodz, Poland.

E-mail: nefro@wp.pl

Released online in J-STAGE as advance publication November 6, 2021. 